\title{
Variação interobservador no diagnóstico histopatologico do carcinoma ductal in situ da mama
}

\author{
Interobserver variation of the histopathologic diagnosis of ductal carcinoma in situ of the breast
}

\author{
Márcio de Almeida Salles ${ }^{1}$, Marco Antonio Rodrigues Freire Matias ${ }^{2}$, \\ Lúcio Márcio Perri de Resende ${ }^{3}$, Helenice Gobbi ${ }^{4}$
}

\section{RESUM0}

\begin{abstract}
Objetivos: fazer avaliação crítica do diagnóstico histopatológico do carcinoma ductal in situ (CDIS) da mama empregando a variação interobservador quanto ao diagnóstico, padrão arquitetural predominante, grau nuclear e grau histológico. Métodos: oitenta e cinco casos com diagnóstico inicial de CDIS foram revisados por um mesmo patologista, especialista em patologia mamária, que selecionou 15 casos para análise interobservador. A análise foi realizada por cinco patologistas e um especialista internacional em patologia mamária, que receberam as mesmas lâminas e um protocolo para classificar as lesões em hiperplasia ductal atípica (HDA), CDIS e CDIS com microinvasão (CDIS-MIC). Caso o diagnóstico fosse de CDIS, os patologistas deveriam também classificá-lo quanto ao padrão arquitetural, grau nuclear e grau histológico. Os resultados foram analisados usando-se concordância percentual e o teste kappa. Resultados: houve grande variação diagnóstica interobservador. Em um caso tivemos todos os diagnósticos, desde HDA, CDIS até CDIS-MIC. Usando o teste kappa para a comparação entre os diagnósticos dos cinco observadores e o especialista internacional obtivemos concordância interobservador mínima $(<0,40)$. Quanto à classificação do CDIS em relação ao padrão arquitetural e ao grau histológico, os valores do teste kappa foram considerados ruins quanto à concordância interobservador. Os melhores resultados foram obtidos na análise da concordância quanto ao grau nuclear, com índices kappa de até 0,80 , considerados como boa concordância. Conclusão: os baixos índices de concordância interobservador no diagnóstico e classificação do CDIS da mama indicam a dificuldade na utilização dos critérios diagnósticos mais empregados na literatura na interpretação destas lesões e a necessidade de treinamento especifico dos patologistas não-especialistas no diagnóstico destas lesões.
\end{abstract}

PALAVRAS-CHAVE: Mama: carcinoma; Mama: carcinoma ductal in situ; Hiperplasia ductal; Histopatologia; Variação interobservador

\section{ABSTRACT}

Purpose: to perform a critical evaluation of the histopathological diagnosis of ductal carcinoma in situ (DCIS) of the breast, through the analysis of interobserver variation related to diagnosis, architectural pattern, nuclear grade, and histological grade. Methods: eighty-five cases with an initial diagnosis of DCIS were reviewed by the same pathologist, specialist in breast pathology, who selected 15 cases for interobserver analysis. The analysis was carried out by five pathologists and an international expert in breast pathology, who received the same slides and a protocol for classifying the lesions as atypical ductal hyperplasia (ADH), DCIS, or ductal carcinoma in situ with microinvasion (DCIS-MIC). If the diagnosis was DCIS, the pathologists should classify it according to the dominant architectural pattern, nuclear grade, and histological grade. The results were analyzed using percent concordance and the kappa test. Results: there was a great interobserver diagnostic variation. In one case we had all diagnoses, from ADH, DCIS to DCIS-MIC. The kappa test for the comparison among the five observers' and the expert's diagnoses showed minimum interobservers' concordance $(<0.40)$. Regarding DCIS classification related to the dominant architectural pattern and the histological grade, the kappa test values were considered poor among the pathologists. The best results were obtained for the nuclear grading, with a kappa index up to 0.80 , considered as good concordance. Conclusion: the low index of interobserver concordance in diagnosis and classification of DCIS of the breast indicates the difficulty in using the most common diagnostic criteria of the literature and the need for specific training of non-specialist pathologists in breast pathology for the diagnosis of these lesions.

KEYWORDS: Breast: neoplasms; Atypical ductal hyperplasia; Breast; Ductal carcinoma in situ; Pathology; Interobserver variation

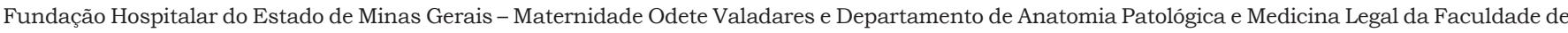
Medicina da Universidade Federal de Minas Gerais

1,2,3 Mastologista da Maternidade Odete Valadares - (Fundação Hospitalar do Estado de Minas Gerais).

4 Professora Adjunto-Doutor da Faculdade de Medicina da Universidade Federal de Minas Gerais.

Correspondência: Márcio de Almeida Salles

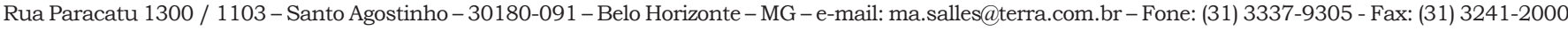

Recebido em: 8/12/2004 Aceito com modificações em: 10/1/2005 


\section{Introdução}

A modificação mais importante no diagnóstico do câncer mamário nas últimas décadas se deveu ao desenvolvimento do rastreamento mamográfico, técnica capaz de detectar carcinomas não palpáveis com diâmetros de apenas alguns milimetros e de identificar lesões ocultas em mulheres assintomáticas. Com os programas de triagem ou rastreamento mamográfico, houve aumento significativo do diagnóstico do carcinoma ductal in situ (CDIS) ${ }^{1,2}$. Com o diagnóstico precoce, surgiram novos desafios para aqueles que convivem com o câncer de mama. Mastologistas e patologistas passaram a lidar com lesões até então pouco freqüentes, referidas como lesões prémalignas ou de risco, as hiperplasias epiteliais e o $\mathrm{CDIS}^{3}$. Embora os programas de rastreamento tenham como base a mamografia, as decisões terapêuticas e estimativas prognósticas dependem do diagnóstico histopatológico, classificação e graduação das lesões. Vários estudos têm analisado a habilidade dos patologistas no diagnóstico e graduação das lesões proliferativas e encontraram concordância diagnóstica baixa ${ }^{3}$. O correto diagnóstico das chamadas lesões pré-malignas tem assumido grande importância após a introdução dos programas de triagem mamográfica, que passaram a detectar aumento expressivo de casos de hiperplasia ductal atípica (HDA) e CDIS ${ }^{2}$.

CDIS é uma proliferação celular anormal com predileção pelas unidades ducto-lobulares terminais da mama, associadas a proeminente envolvimento de ductos verdadeiros e alto risco de recorrência local sem adequado tratamento ${ }^{3,4}$. O CDIS é uma lesão heterogênea sob o ponto de vista histológico e pode conter um espectro de padrões arquiteturais em um mesmo caso ${ }^{5}$. O diagnóstico, em algumas situações, pode ser difícil para o patologista, principalmente quando há necessidade de diagnóstico diferencial entre HDA e o CDIS de baixo grau, ou nos casos de CDIS com suspeita de microinvasão (CDIS-MIC) ${ }^{3,4,6}$.

A variação diagnóstica entre HDA versus CDIS e entre CDIS versus CDIS-MIC tem importantes implicações clínicas. A conduta para uma paciente com HDA poderá ser apenas o acompanhamento clínico e mamográfico. Para as pacientes com CDIS, as opções terapêuticas variam desde ressecção ampla ou lumpectomia, associada ou não à radioterapia, até mastectomia com abordagem axilar ${ }^{7-11}$. Por isso, o diagnóstico histopatológico acurado tem sido considerado um dos parâmetros mais importantes para a decisão terapêutica, sendo o papel do patologista fundamen- tal no tratamento das pacientes ${ }^{6}$. Assim, o treinamento no reconhecimento dos padrões morfológicos do espectro de lesões proliferativas epiteliais mamárias ainda é o principal instrumento do patologista no diagnóstico dessas lesões.

O objetivo deste estudo foi avaliar a variação interobservador no diagnóstico histopatológico do CDIS entre patologistas.

\section{Métodos}

Foi realizado estudo observacional do tipo coorte transversal. Foram revistos 85 casos com diagnóstico de CDIS provenientes do Serviço de Mastologia da Maternidade Odete Valadares e do Hospital das Clínicas da Universidade Federal de Minas Gerais. Selecionaram-se 15 casos com diagnóstico inicial de CDIS que mostraram alguma discrepância entre o diagnóstico inicial e após revisão histopatológica realizada pela mesma patologista com treinamento especializado em patologia mamária. Estes 15 casos foram reavaliados pelo Professor David L. Page, da Vanderbilt University, EUA, renomado especialista em patologia mamária, para confirmação dos diagnósticos histopatológicos.

Os critérios diagnósticos utilizados foram descritos por ele e seus colaboradores e publicados em vários trabalhos sobre este tema ${ }^{3,12-14}$. O CDIS é uma proliferação epitelial de células atipicas, que envolve completamente dois "espaços" ou ductos, ou medem em conjunto mais de 2 a $3 \mathrm{~mm}$. A HDA é diagnóstico de exclusão e deve ser feito quando elementos que definem citologicamente e histologicamente CDIS de baixo grau estão presentes, mas envolvem incompletamente dois "espaços" ou ductos, ou medem em conjunto menos de 2 a $3 \mathrm{~mm}$. O diagnóstico de microinvasão é feito quando existe um CDIS predominante, com um ou mais focos de carcinoma invasor de dimensão máxima de $3 \mathrm{~mm}$ além da membrana basal epitelial, presente no tecido conjuntivo não especializado ou extra-lobular. Os diagnósticos dessa revisão especializada foram considerados como "padrão" na análise da variação diagnóstica interobservador. Dentre os 15 casos analisados, dois foram considerados pelo especialista internacional como HDA e treze casos de CDIS, sendo quatro casos de CDIS de grau histológico baixo, dois casos de CDIS de grau intermediário e sete casos de CDIS de grau alto.

Para a análise interobservador, foram convidados cinco patologistas da comunidade, cujos laboratórios foram responsáveis pelos diagnósti- 
cos iniciais. Estes patologistas relataram em questionário especifico, preenchido antes do estudo, terem experiência em patologia mamária e tempo de atuação como patologistas variando entre quatro a 17 anos. Eles afirmaram analisar entre 250 e 1500 espécimes mamários por ano, cada. As mesmas lâminas dos 15 casos foram enviadas para os cinco patologistas juntamente com um protocolo específico, para que as lesões fossem classificadas quanto ao diagnóstico como: HDA, CDIS ou CDIS-MIC. Se o diagnóstico fosse de CDIS ou CDIS-MIC, deveria ser classificado quanto ao padrão arquitetural predominante em: comedocarcinoma (COME), cribriforme (CRIB), papilar (PAPI), micropapilar (MIPA) ou sólido (SOLI); quanto ao grau nuclear, em baixo, intermediário ou alto grau; quanto ao grau histológico, em baixo, intermediário ou alto grau. O patologista deveria classificar as lesões de acordo com os critérios usados na rotina de seu laboratório.

Para a análise da variação interobservador do diagnóstico histopatológico dos 15 casos de CDIS selecionados foram utilizados a concordância percentual e o teste kappa, interpretado de acordo com os índices sugeridos por Jekel et al. ${ }^{15}$ : $<0,20$ (desprezivel), 0,21-0,40 (minima), 0,41-0,60 (ruim), 0,61-0,80 (boa), 0,81-1,0 (excelente). O teste kappa tem sido utilizado para avaliar a concordância entre dois observadores. Admite-se que alguma proporção do grau de concordância surja simplesmente ao acaso. Emprega-se o teste kappa para tentar minimizar a influência do acaso na interpretação dos dados de concordância ${ }^{15}$.

Este trabalho foi aprovado pelo Comitê de Ética em Pesquisa da Universidade Federal de Minas Gerais (parecer ETIC 103/01).

\section{Resultados}

Quanto ao diagnóstico, observamos concordância completa entre os cinco observadores e o especialista internacional em apenas 2 dos 15 casos (13,3\%). Em seis casos, houve concordância entre quatro observadores e o especialista internacional (40,0\%). No caso " 1 " tivemos todos os diagnósticos possiveis, desde HDA, CDIS até CDIS-MIC. Um observador tendeu para benignidade, diagnosticando HDA em 7 casos, ao passo que o especialista internacional o fez em apenas dois casos. Outro observador tendeu para malignidade, diagnosticando CDIS-MIC em seis casos, ao passo que o especialista não fez esse diagnóstico em nenhum dos 15 casos (Figura 1).

Os valores do teste kappa comparando o observador A (especialista internacional) com os demais observadores (B, C, D, E e F), variaram de 0,09 a 0,39, indices considerados mínimos.

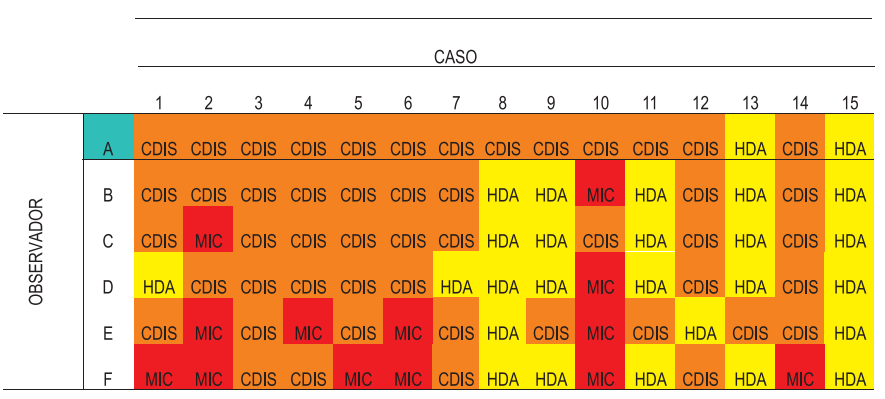

HDA: Hiperplasia ductal atípica; CDIS: Carcinoma ductal in situ; MIC: CDIS com microinvasão.

Figura 1 - Variação interobservador do diagnóstico de 15 casos inicialmente diagnosticados como carcinoma ductal in situ, realizado por cinco patologistas (observadores B a F) comparados aos diagnósticos de um especialista internacional em patologia mamária (observador $\mathrm{A}$ ).

Quanto ao padrão arquitetural predominante observamos concordância diagnóstica completa entre os cinco observadores em apenas um caso $(7,7 \%)$ (Figura 2). O teste kappa comparando o observador A (especialista internacional) com os demais observadores $(\mathrm{B}, \mathrm{C}, \mathrm{D}, \mathrm{E}$ e F), mostrou índices variando de 0,32 a 0,43 , considerados mínimo e ruim, respectivamente.

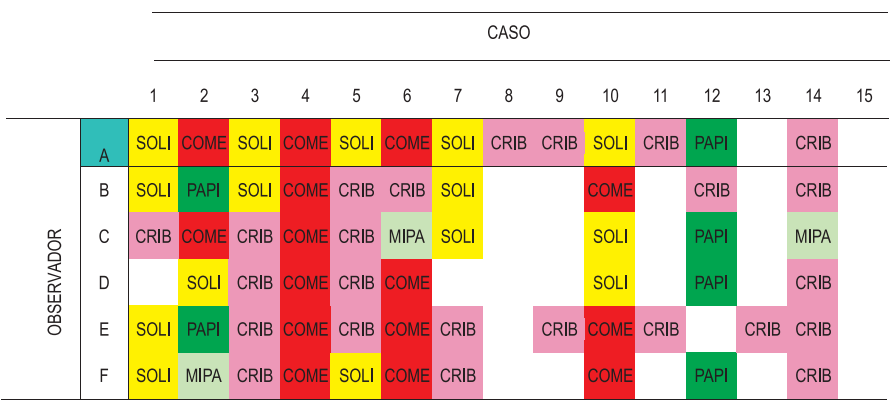

SOLI: sólido; CRIB: cribriforme, COME: comedo; PAPI: papilar MIPA: micropapilar. Os quadros em branco se referem aos casos em que não se fez diagnóstico de CDIS.

Figura 2 - Variação interobservador quanto ao padrão arquitetural predominante em 15 casos inicialmente diagnosticados como carcinoma ductal in situ, realizado por cinco patologistas (observadores B a F) comparados aos diagnósticos de um especialista internacional em patologia mamária (observador A).

Quanto ao grau nuclear, observamos concordância diagnóstica completa entre os cinco observadores e o especialista internacional em quatro casos (30,8\%) (Figura 3). O teste kappa para a comparação entre o observador A (especialista internacional) e os demais observadores (B, C, D, E e F) mostrou índices de 0,24 a 0,74 , considerados mínimo e bom, respectivamente. 


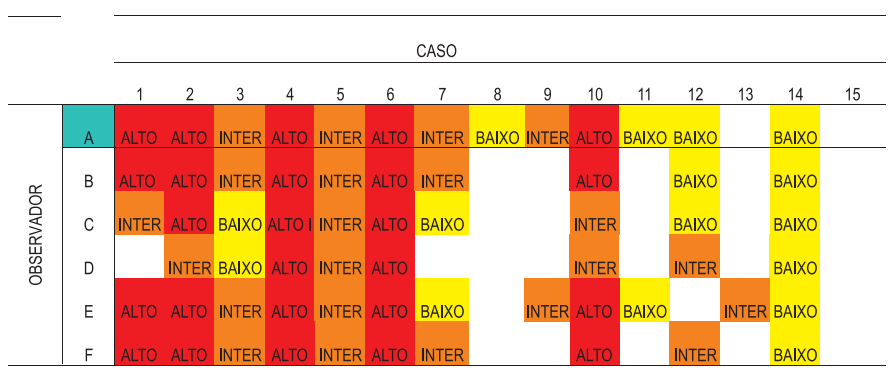

INTER: Grau intermediário.

Os quadros em branco se referem aos casos em que não se fez diagnóstico de CDIS.

Figura 3 - Variação interobservador quanto ao grau nuclear em 15 casos inicialmente diagnosticados como carcinoma ductal in situ, realizado por cinco patologistas (observadores $\mathrm{B}$ a F) comparados aos diagnósticos de um especialista internacional em patologia mamária (observador $\mathrm{A}$ ).

Quanto ao grau histológico, houve concordância diagnóstica completa entre os cinco observadores e o especialista internacional em três casos (23,1\%) (Figura 4). Os índices do teste kappa para a comparação entre o observador A (especialista) e os demais observadores (B, C, D, E e F) variaram de 0,03 a 0,52, indices considerados desprezivel e mínimo, respectivamente.

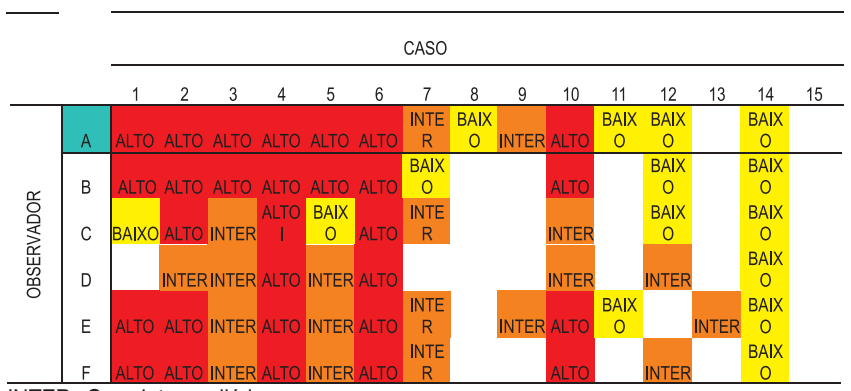

INTER: Grau intermediário.

Os quadros em branco se referem aos casos em que não se fez diagnóstico de CDIS.

Figura 4 - Variação interobservador quanto ao grau histológico predominante em 15 casos inicialmente diagnosticados como carcinoma ductal in situ, realizado por cinco patologistas (observadores B a F) comparados aos diagnósticos de um especialista internacional em patologia mamária (observador A).

\section{Discussão}

Nas ultimas duas décadas, devido ao uso crescente do rastreamento mamográfico, nos deparamos cada vez mais com os diagnósticos das lesões epiteliais proliferativas e do CDIS da mama. Tornam-se necessários diagnósticos precisos para uma adequada abordagem terapêutica, que será possivel com bom entendimento dos padrões morfológicos destas lesões. Visando uma análise crítica dos diagnósticos histopatológicos, comparamos o diagnóstico de cinco patologistas da comunidade com os de um especialista de reno- me internacional com vasta experiência e numerosas publicações nesta área e que foi considerado o diagnóstico padrão.

Os nossos dados mostraram grande variação diagnóstica interobservador. A variação nos diagnósticos das lesões proliferativas intraductais traz grandes dificuldades e dúvidas para os mastologistas na abordagem terapêutica das pacientes. No caso 1 do nosso estudo os diagnósticos variaram de HDA até CDIS-MIC. Neste caso específico, dependendo do diagnóstico, o tratamento poderia variar de simples acompanhamento até mastectomia com abordagem axilar.

Outros autores também compararam a habilidade do patologista em classificar as lesões proliferativas pré-malignas da mama. Rosai ${ }^{16}$ selecionou dezessete casos de lesões epiteliais proliferativas da mama e submeteu-os à avaliação por cinco patologistas experientes em patologia mamária, com o objetivo de avaliar os critérios usados por Page e Anderson ${ }^{3}$ para o diagnóstico de HDA vs CDIS. Não houve concordância diagnóstica entre os cinco observadores em nenhum caso. Houve concordância diagnóstica entre quatro patologistas em três casos (18\%). Em seis casos (33\%), houve todos os diagnósticos possíveis, variando de hiperplasia sem atipia até carcinoma in situ (CIS). Enquanto um patologista tendia para benignidade, outro tendia para malignidade nos seus diagnósticos. Schnitt et al. ${ }^{17}$ fizeram um estudo submetendo 24 casos de lesões proliferativas à avaliação por seis patologistas, que deveriam classificá-las em hiperplasia sem atipia, hiperplasia atípica ou CIS. Antes de iniciar a reavaliação destas lesões, os patologistas foram treinados empregando-se os critérios de Page et al. ${ }^{18}$. Obteve-se concordância diagnóstica completa entre os seis patologistas em quatorze casos (58\%), concordância diagnóstica entre cinco ou mais patologistas em dezessete casos $(71 \%)$ e concordância diagnóstica entre quatro ou mais patologistas em 22 casos (92\%). Nenhum patologista mostrou tendência para diagnósticos benignos ou malignos em relação aos demais. Estes resultados mostram que o treinamento e o uso de critérios bem definidos para classificar as lesões epiteliais proliferativas da mama fazem melhorar os índices de concordância diagnóstica interobservador. O treinamento para o reconhecimento dos padrões morfológicos deste difícil espectro de lesões epiteliais proliferativas mamárias continua sendo o maior desafio para os patologistas e considera-se a principal forma de minimizar as diferenças no diagnóstico destas lesões ${ }^{17}$.

Alguns autores ${ }^{19}$ relataram que o maior problema em se determinar o padrão arquitetural 
predominante é a falta de reprodutibilidade. Isso se deve principalmente à heterogeneidade arquitetural e à presença em uma mesma lesão de diferentes padrões arquiteturais que podem estar unidos uns aos outros. Um baixo índice kappa, 0,23 (23\%), foi obtido na avaliação do padrão arquitetural predominante de casos de CDIS no programa de qualidade do U.K. National EQA ${ }^{20}$.

Em nossa avaliação interobservador do grau nuclear, cinco de quinze casos foram considerados CDIS de alto grau pelo especialista internacional e tiveram concordância diagnóstica total com três observadores. Não foi observado o mesmo índice de concordância diagnóstica em lesões de CDIS de grau intermediário e baixo, porém não houve variação de mais de um grau entre os observadores e o padrão. O teste kappa entre o padrão e três observadores foi maior que 0,60 (60\%), considerado de boa concordância diagnóstica interobservador. Bethwaite et al. ${ }^{21}$ submeteram 25 casos de CDIS à avaliação de onze patologistas, que deveriam graduar as lesões utilizando os critérios de Holland et al. ${ }^{22}$ e de Silverstein et al. ${ }^{23}$. Observaram concordância diagnóstica interobservador maior quando se usava a classificação Van Nuys, com índice kappa de até 0,66 (66\%). Em um estudo ${ }^{24}$ para avaliação do grau nuclear, seis patologistas analisaram 125 casos de CDIS. Encontraram concordância diagnóstica interobservador com indice de kappa variando de $0,39(39 \%)$ a $0,61(61 \%)$. Semelhante ao nosso estudo, quando havia discordância entre os observadores, em nenhum caso havia variação maior que um grau. Comparando os nossos dados com os desses dois estudos, observamos que a concordância diagnóstica interobservador é maior nos casos de CDIS de alto grau.

Scott et al. ${ }^{14}$ relataram concordância diagnóstica interobservador quanto ao grau histológico em $94 \%$ dos casos de CDIS. Bethwaite et al. ${ }^{21}$ encontraram concordância diagnóstica interobservador na graduação histológica do CDIS com indice de kappa de 0,44 (44\%) e 0,66 (66\%), usando critérios de Holland et al. ${ }^{22}$ e de Silverstein et al. ${ }^{23}$, respectivamente. Diferentemente, em nosso estudo, solicitamos aos patologistas que classificassem e graduassem as lesões utilizando os sistemas que empregavam na rotina de seus laboratórios e não um critério específico com treinamento prévio. Os baixos índices de concordância diagnóstica que observamos podem ser devidos à utilização de diferentes critérios para diagnóstico, à dificuldade diagnóstica inerente à complexidade dessas lesões e ainda à pouca experiência de alguns examinadores com esse grupo de lesões.
Nossos resultados e os da literatura apontam a necessidade de se padronizarem internacionalmente, por meio de uma classificação consensual, os critérios para o diagnóstico histológico das hiperplasias epiteliais e do CDIS da mama e, também, a necessidade de treinamento específico dos patologistas no diagnóstico dessas lesões.

Em base dos resultados do nosso estudo, realizado com patologistas que atuam na prática clinica diária, avaliando lesões mamárias proliferativas pré-invasivas de difícil diagnóstico diferencial, com implicações terapêuticas diferentes, recomendamos reavaliação diagnóstica por um segundo patologista com treinamento especializado em patologia mamária nos casos de hiperplasia ductal atípica, carcinoma ductal in situ de baixo grau e carcinoma ductal in situ de alto grau com microinvasão. A revisão histopatológica especializada pode diminuir os impactos de tratamentos inadequados e desgastes pessoais e financeiros.

Agradecimentos: Ao Dr. David L. Page, da Vanderbilt University, Nashville, EUA, que gentilmente aceitou participar deste trabalho como revisor dos 15 casos. Aos médicos patologistas da nossa comunidade que também nos prestigiaram com a participação neste estudo: Dr. Agostinho Pinto Gouvêa, Dra. Daniela Savi, Dr. Marco Aurélio Figueiredo, Dr. Ramão Tavares Neto e Dr. Rodrigo Assis de Paula.

\section{Referências}

1. Ernster VL, Barclay J, Kerlikowske K, Grady D, Henderson C. Incidence and treatment for ductal carcinoma in situ of the breast. JAMA. 1996; 275:913-8.

2. Fentiman IS. Ductal carcinoma in situ. In: Fentiman IS, editor. Detection and Treatment of Breast Cancer. $2^{\text {nd }}$ ed. London: British Library; 1998. p. 217-33.

3. Page DL, Anderson TJ. Diagnostic histopathology of the breast. $1^{\text {st }}$ ed. Edinburgh: Churchill Livingstone; 1987. p. 120-92.

4. Ministério da Saúde. Instituto Nacional do Cancer. Diagnóstico histopatológico e citopatológico das lesões da mama. Rio de Janeiro: CONPREV; 2002.

5. Lennington WJ, Jensen RA, Dalton LW, Page DL. Ductal carcinoma in situ of the breast: heterogeneity of individual lesions. Cancer. 1994; 73:11824.

6. Ellis IO, Elston CW, Poller DN. Ductal carcinoma in situ. In: Elston CW, Ellis IO, editors. The 
breast. Edinburgh: Churchill Livingstone; 1998; p. 249-82.

7. Tavassoli FA, Man Y. Morphofunctional features of atypical intraductal hyperplasia, and various grades of intraductal carcinoma. Breast J. 1995; 1:155-62.

8. The Consensus Conference on the Treatment of In Situ Ductal Carcinoma of the Breast. Breast J. 2000; 6:4-13.

9. Silverstein MJ, Lagios MD, Groshen S, et al. The influence of margin width on local control of ductal carcinoma in situ of the breast. N Engl J Med. 1999; 340:1455-61.

10.Consensus Conference on the Classification of Ductal Carcinoma In Situ. Hum Pathol. 1997; 28:1221-5.

11.Silverstein MJ, Barth A, Poller DN, et al. Ten-years results comparing mastectomy to excision and radiation therapy for ductal carcinoma in situ of the breast. Eur J Cancer. 1995; 31A:1425-7.

12.Page DL, Lagios MD. Pathology and clinical evolution of ductal carcinoma in situ (DCIS) of the breast. Cancer Lett. 1994; 86:1-4.

13.Page DL, Rogers LW. Combined histologic and cytologic criteria for the diagnosis of mammary atypical ductal hyperplasia. Hum Pathol. 1992; 23:1095-7.

14. Scott MA, Lagios MD, Axelsson K, Rogers LW, Anderson TJ, Page DL. Ductal carcinoma in situ of the breast: reproducibility of histological subtype analysis. Hum Pathol. 1997; 28:967-73.

15.Jekel JF, Elmore JG, Katz DL. Epidemiologia, bioestatistica e medicina preventiva. Porto Alegre: Artmed; 1999. p. 99-112.
16.Rosai J. Borderline epithelial lesions of the breast. Am J Surg Pathol. 1991; 15:209-21.

17.Schnitt SJ, Connolly JL, Tavassoli FA, et al. Interobserver reproducibility in the diagnosis of ductal proliferative breast lesions using standardized criteria. Am J Surg Pathol. 1992; 16:1133-43.

18.Page DL, Dupont WD, Lowell WR, Rados MS. Atypical hyperplastic lesions of the female breast. A longterm follow-up study. Cancer. 1985; 55:2698-708.

19. Shoker BS, Sloane JP. DCIS grading schemes and clinical implications. Histopathology. 1999; 35:393400.

20.Sloane JP, Ellman R, Anderson TJ, et al. Consistency of histopathological reporting of breast lesions detected by screening: findings of the U.K. National External Quality Assessment (EQA) Scheme. U. K. National Coordinating Group for Breast Screening Pathology. Eur J Cancer. 1994; 30A:1414-9.

21.Bethwaite P, Smith N, Delahunt B, Kenwright D. Reproducibility of new classification schemes for the pathology of ductal carcinoma in situ of the breast. J Clin Pathol. 1998; 51:450-4.

22.Holland R, Peterse JL, Millis RR, et al. Ductal Carcinoma in situ: a proposal for a new classification. Semin Diagn Pathol. 1994; 11:167-80.

23. Silverstein MJ, Poller DN, Waisman JR, et al. Prognostic classification of breast ductal carcinoma in situ. Lancet. 1995; 345:1154-7.

24.Sneige N, Lagios MD, Schwarting $R$, et al. Interobserver reproducibility of the Lagios nuclear grading system for ductal carcinoma in situ. Hum Pathol. 1999; 30:257-62. 